\title{
The Politics of Chaos: Local Knowledge versus Global Theory
}

I $\mathrm{N}$ the late twentieth century, local knowledge has come into its own. Across a wide spectrum of contemporary theory-in philosophy, feminism, literary criticism, and cultural analysis-theorists insist that local variations must be respected in their own right and not simply incorporated into global schemes. The issue is made more complex by the shifting meanings that "local" and "global" carry in these arguments. In critical theory, for example, "global" is understood to mean not only cultural systems considered as a whole but any theory that subsumes particular texts or phenomena into a universal explanation. Marxism, relativity theory, and grammar are global theories in this sense. Similarly, "local" connotes both a small subsection of a geopolitical area, such as the western coast of Brittany, and particular textual or cultural sites that resist assimilation into the generalizations of a universal theory.

The conflation of geopolitical with theoretical connotations is significant, for it signals a growing feeling that totalizing theories should be discredited because they are associated with oppressive political structures. Particularly important here are Foucault's archaeological analyses of the totalizing theories of the Enlightenment, from grammar to biology to penology, and their complicity with totalitarian political practices (1970, 1973, I977). By implication, local knowledge has become associated with liberation. I shall look 
critically at these assumptions, arguing that local knowledge does not necessarily lead to liberation, any more than generalizing theories always lead to oppression.

The new scientific paradigms, especially the science of chaos, have been seen as carrying the case for local knowledge into the physical sciences. This argument is also suspect, for reasons I will discuss shortly. It is true, however, that the sciences of chaos make the local/ global relation problematic in a way it was not in older paradigms. With the onset of chaos, different levels tend to act in different ways, so that locality intrudes itself as a necessary descriptive feature, defeating totalization. To illustrate this process for fractals, Mandelbrot asks a question that looks as if it should have a straightforward, factual answer: How long is the coastline of Britain? (1983: 25-33). As I intimated earlier, the question is more devious than it appears, because the answer is scale-dependent. If we use a mile-long ruler to measure the coastline, we get a shorter answer than we do if we use a yardstick, for the mile ruler cuts across irregularities that the yardstick measures around. If we use an inch ruler the answer is still longer, because small pebbles are measured around; and if a micrometer is used, even irregularities within a single pebble count. In fact, Britain's coastline continues to grow without limit as the ruler scale decreases, at least down to molecular scales. Unless the length of the ruler is specified, the question cannot be accurately answered.

The example illustrates how questions of scale are foregrounded in the new paradigms, in contrast to Euclidean geometry and Newtonian mechanics. In these older paradigms the idea that one could get different answers with different scales does not appear. It is not quite correct to say they make global statements that are considered true at every level, because their globalizing approaches are so complete that the system is not conceived as having levels in any meaningful sense. In Euclidean geometry, for example, it does not matter whether an isosceles triangle is twice as large or two hundred times as large as another triangle of the same shape; whatever the scale, Euclidean geometry states, the three sides of similar triangles will be in the same proportion to one another. Fractal geometry does not challenge this assertion as such. Rather, it shifts the focus to complex irregular forms, for which scale appears as an important con- 
sideration and movement between scales is highly nontrivial. Similarly, Newtonian mechanics applies the same partial differential equations whether the object is a golf ball or a planet; in either case the masses move through time according to uniform laws of motion. However, when the object has a complex internal structure consisting of distinct local levels, such as a fluid in turbulent flow, the length of the scale is critical because different portions of the fluid move at different speeds and with different kinds of motions. Under these conditions, Newtonian-based calculations are unmanageable for even a few points, and unthinkable for the thousands or millions it would take to model the system.

It is important to understand that chaos theory does not renounce globalization. Rather, chaos theory achieves globalization in a different way, by correlating movements from one level to another. We saw in chapter 7 that a standard method for analyzing complex systems is to look for shapes or configurations having the property of self-similarity. Self-similarity is an essential characteristic because it is only when complex systems have this property that scales of different lengths can easily be correlated. The distinction between the classical and new paradigms is not, then, that one globalizes and the other does not, but that one is scale-invariant and the other is not. In this respect chaos theory extends the lessons of quantum physics and relativity theory. After Einstein, Bohr, Planck, and Schrödinger, physicists learned to specify that Newtonian equations applied only at nonrelativistic speeds and to distances and masses where quantum effects could be ignored. Quantum mechanics and the special theory of relativity thus introduced scale considerations-but only for the very small and the very fast. Chaos theory, by contrast, teaches that scale is generally important for complex systems, even at nonrelativistic speeds and for macroscopic dimensions.

Chaos theory has also changed expectations about proportionality between cause and effect. In classical paradigms, a small cause is generally associated with a small effect. If the angle between the sides of a triangle changes by one degree, for example, the resulting form is only a little different from its predecessor. In a complex system, by comparison, small changes in the iterative formulae or initial conditions can result in very large changes in the final state. As we saw earlier, this complex relation between the system's behavior and 
its analytical description is a function of its infinitely fine-grained structure, with symmetries on one level giving way to symmetries on the next, and so on down. The large number of coupling points between levels means that tiny initial changes can quickly be magnified and brought up to macroscopic expression. The sense that movement between the local and global is highly nontrivial thus applies both to levels within a system and to the correlation between the system's physical properties and its analytical description.

In the problematic relation chaos theory posits between description and behavior, it comes close to the critique of global theories within the human sciences. Consider again the question of Britain's coastline. In addition to demonstrating that the answers to such questions are scale-dependent, Mandelbrot shows that they have a political dimension. Portuguese encyclopedias, for example, report that the boundary Portugal shares with Spain is longer than Spanish encyclopedias say it is, by as much as 20 percent. Mandelbrot conjectures that as a small country, Portugal is more concerned than Spain is with the length of its borders and consequently uses a smaller scale to measure them. The example goes to the heart of the objection to global theories within critical theory: to what extent are global theories social and linguistic constructions, inventing the reality they purport to describe? If the answer is "entirely, or nearly so," then the political and ideological functions global theories serve should be the focus of our inquiry, rather than the validity of the theories themselves.

The critique of global theories, then, proceeds on two counts. In the first instance, global theory is questioned because it is not applicable to complex systems. Clifford Geertz, for example, insists on the superiority of local knowledge over global theory because he regards cultures as complex semiotic systems organized around particular local sites (1973: 3-32). In Geertz's view, when one talks about culture in general, one misses the very essence of what one hopes to understand, for meaning cannot be separated from the particular organization of signs that characterizes a given site. In the second instance, globalizing theories are rejected because they are constructions that serve the vested interests of particular classes or power structures. Local knowledge is superior to global theory be- 
cause, far from reinscribing differences that have marked oppressed people as inferior, it reveals fissure lines marking the interests of the oppressed as different from those of the people in power.

No one would debate the importance of this work. To see the oppression that global theories have too often reinforced or enabled, it is enough to remember how a few European cultures have been equated with mankind; how mankind has been equated with humankind; how humankind has been equated with intelligent consciousness. As only one example of the powerful analyses to which the privileging of local knowledge gives rise, consider Mary Poovey's article (1986) on the nineteenth-century debate within the medical community over whether ether or chloroform should be the anaesthetic of choice. Poovey demonstrates that this seemingly objective question in fact reveals fissure lines that follow the division of power between male midwives and surgeons, both fighting to establish their legitimacy against female midwives and to capture the lucrative practice associated with medical intervention in the birth process. The anaesthetized body of the woman is the blank space that each group textualizes according to its interests. Clearly, these totalizations do not reveal the experiences actual women had, much less the universal truth about such experiences; rather, they are the constructions that the two sides found expedient for their purposes. If either the medical discourse or its image of woman is seen as monolithic, the rifts that mark the play of power are hidden from view. In this instance as in countless others, attending to difference opens discourse to the kind of ideological analysis that can unmask totalizations for the political constructions they are.

Given the power and importance of this method, it is easy to see how the case for difference can be viewed as irrefutable. Under these circumstances, who would want to criticize local knowledge or endorse global theory? If we regard the case as closed, however, we paradoxically blind ourselves to differences within the wide-ranging debate about the local and global in contemporary thought. In the arguments that follow, I try to open this play of difference by drawing distinctions between what "local" and "global" mean in the sciences of chaos and in critical theory. In the new scientific paradigms, the global subsumes the local, but at the price of reconceptualizing 
the global as constituted by locality. Within critical theory, the claims of the local are expanded until the local itself becomes a new kind of globalizing imperative.

These two impulses mirror each other, for in the sciences of chaos the global is localized, and in critical theory the local is globalized. If the local and global turn themselves inside out when pushed to extremes, how can their conflicting claims be adjudicated? In searching for ways to articulate them together, $I$ argue that it is wrong to assume that global theory is always politically more coercive than local knowledge. For example, the abolitionist argument that slaves had souls was a global theory about human nature; yet it could hardly be said to be more coercive than local knowledge about slaves in the South. ${ }^{1}$ The example demonstrates that the case for local knowledge, to be meaningful, has to be historically specific. To argue that local knowledge is always better than global theory is, in effect, to create a global theory of local knowledge. Conversely, to ignore the claims of the local is to regress to totalizing theories that have historically been associated with repression. How to find a balance? The question is inherently paradoxical, for to answer it one must put forward generalizations, yet generalizations are precisely what are at issue. The question is also highly charged, for of all the issues energized by the new science, it is the most inescapably political.

Why should there be a balance? This prior question goes to the heart of the issue, for the local/global dialectic is deeply tied up with the long-standing debate between the physical and human sciences about the extent to which reality is a social construction. If there is only epistemology and no ontology, universals within physical theories reflect only how we perceive, never reality itself. In this view, there is no difference between the second law of thermodynamics and the doctrine of white supremacy; both are social and political constructions that serve the interests of specific groups of people at specific times. In my view, this statement is both true and false. It is true in the sense that all knowledge, including the second law of thermodynamics, is representational. It is false in the sense that some representations of the physical world can express universals that are

'My thanks to Donald N. McCloskey for suggesting this example to me. 
true at many locales. As Michel Serres puts it in Hermes (1982a), "Entropy always increases, whatever the latitude and whoever the ruling class." Finding a balance implies finding a way to say that entropy always does increase, at least in this universe, and also that entropy is a social and historical construction that has meant different things to different people for different reasons. If I seem more critical of local knowledge than of global theory, it is because in the last decade the ideology of local knowledge has been too often assumed and too little examined. My purpose is not, however, to criticize local knowledge as such, but to explore its limits so as to allow also for the possibility of global theory.

The ideological freight carried by the valorization of local knowledge is explicit in Jean-François Lyotard's conclusion to The Post-

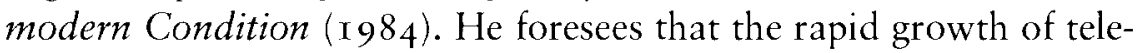
tronics in highly developed societies will further consolidate power in the hands of the elite who have access to data banks. He implies that this trend can be countered by the emergence within science of fractal geometry, quantum mechanics, catastrophe theory, and Gödel's theorem. Grouping these disparate theories under the label "paralogy," Lyotard suggests that they will let us "wage a war on totality; let us be witnesses to the unpresentable; let us activate the differences and save the honor of the name" (p. 82). Here, then, the new paradigms are enlisted under the banner of local knowledge to neutralize the totalizing potential of modern information technology. The argument that "paralogy" can rescue postmodern culture from totalitarianism is ill founded for several reasons. It is akin to social Darwinism, in that it confuses scientific theories with social programs. But even if this were not so, the argument still ignores the fact that the global is not absent from these theories, only redefined by them. The endorsement of local knowledge within contemporary science cannot be the panacea Lyotard imagines. It is true that the new paradigms recognize the importance of scale, and therefore of locality. But it is also true that these changes are located within disciplines committed to universal theory. These disciplinary commitments to universality inform the theories as surely as the theories help to form the disciplines.

The universalizing impulse within chaos theory is hard to miss. Surely chaos theory would not have attracted the attention it has if it 
simply confirmed the obvious, that chaos is disordered. No: what makes it noteworthy is the discovery of order in the midst of disorder. In Feigenbaum's study of iterative functions (1980), the result that makes his work ground-breaking is his discovery that chaotic systems, despite their inherent unpredictability, share certain universal characteristics. The name he chooses to describe his discovery"universality theory" - clearly conveys this message. The article on strange attractors by Crutchfield, Farmer, Packard, and Shaw in Scientific American (I986) is another case in point. For Crutchfield and his collaborators, "chaos" connotes a patterned unpredictable trajectory in phase space. If a system is not a strange attractor-if it is simply disordered and nothing more-they consider it a "featureless blob" of no interest (p. 56). Even Mandelbrot (1983), though his rhetoric celebrates disorder, looks for the symmetry groups that will allow chaos to be understood as iterative extensions of regular forms. Kenneth Wilson's Nobel Prize work (I 983) on renormalization groups in quantum field theory has the same goal, to find the symmetry operators that permit analytical solutions to chaotic systems. As these scientists use "chaos," it connotes not the unpredictable aspects of disordered systems but their universal characteristics. To see chaos theory as the antidote to totalization is to ignore this thrust toward universality.

Other instances of Lyotard's "paralogy," such as quantum mechanics, are similarly misrepresented when they are enlisted in the cause of local knowledge. Since about I 960 the most active work in quantum mechanics has been the attempt to combine it with relativity theory to create a grand unified field theory. Far from endorsing local knowledge, grand unified theory aims to explain all material interactions in terms of the four basic forces of the universe (Parker, I986; Feynman, I986). How deeply the universalizing impulse is felt within contemporary science is also apparent in the recent work on supersymmetry, which promises to synthesize the four dimensions of spacetime with electricity and magnetism to create models of the universe ranging from ten to twenty-six dimensions (depending on what assumptions are made) capable of explaining all known physical fields through a single theory (Davies, I984). To instance quantum mechanics as an example of local knowledge, then, is to skew the way it is used in its scientific context. 
At the same time, the emphasis on locality within the new paradigms has changed the way the global is conceived. The essential concept in this changed view is self-similarity. Many complex forms have smaller parts that are shaped like the object-a twig reproduces the shape of a branch, and a branch reproduces the shape of a tree. In these instances the whole and the parts are related through self-similarity. Self-similarity has made it possible to encode the information contained in complex forms in new ways. Michael Barnsley's recent work on fractals, for example, starts with an approximation of the desired global shape-say, the rough outline of a fern (Barnsley et al., I 986; Peterson, 1987). Each frond coming out from the fern's central stem is shaped like the fern. These fronds can be sketched through a series of reducing and deforming transformations (known as affine transformations) that operate upon the fern's global shape to create the individual fronds. The computer re-creation can be as detailed as desired. Individual leaflets on a frond, which are shaped like the fern, can also be generated through affine transformations; so can smaller irregularities on the leaflets, and yet smaller variations in these irregularities. In this way, increasingly detailed structures are obtained from the same initial bits of information. The method has considerable generality and can be used to recreate such diverse natural forms as landscapes, capillaries, clouds, and galaxies. It allows complex images, which would normally require several million bits of information to describe, to be encoded through the few iterative formulae used to generate the self-similar shapes and carry them through the transformations. Some researchers predict that in a few years, movies could be transmitted from computer to computer by this method (Peterson, 1987: 283).

As Mitchell Feigenbaum and Benoit Mandelbrot have pointed out, such methods have important epistemological implications. ${ }^{2}$ In the Newtonian paradigm, objects moving through time are modeled as trajectories that can be broken into arbitrarily small intervals (this is essentially the basis for calculus). As these intervals approach zero,

"I am indebted throughout this discussion to the presentations by Mitchell Feigenbaum, "Attempts at the Analytic Description of Complex Phenomena," and Benoit Mandelbrot, "Fractals: Complex Relationships in Mathematics, the Sciences and the Arts," at the conference "Analyzing the Inchoate," Cornell University, April I 6 , 1987. 
they are added together to get the trajectory as a whole. Underlying this method is the common-sense perception that movement through time can be equated with the movement of an object along a predictable path, such as the parabolic arc of a baseball as it comes off the bat. However, if the moving shape is complex in the sense of being composed of multiple levels acting in different ways, the calculations become unmanageable very quickly, because each of the levels has to be advanced through time individually. In these cases, it is much more economical to describe the transformation rules that govern the evolution of the iterative formulae through time. The shape itself, moreover, is no longer conceived as a mass of points, but as the formulae used to generate and randomize the self-similar forms that compose the various levels of the object. In effect, the underlying epistemology has changed radically, for the system no longer is conceived as masses of points moving along a predictable path, but is seen as the evolution of the internal structural principles that describe the propagation of self-similar symmetries.

Consider how this change in epistemology would affect traditional paradigms within the human sciences. Newton's conception of objects as masses of points is analogous to Hobbes's vision of society as a group of autonomous individuals, and to Adam Smith's representation of the economy as a collection of competing customers. In these conceptions, the individual units that collectively constitute the global system are considered to be elemental points acting in accord with general laws. One makes the transition from the local to the global by applying general laws to masses of individual units, and one achieves movement through time by adding together the individual motions to arrive at a resultant. Now compare these classical assumptions to those Foucault uses in his archaeologies. Foucault considers the individual not as an autonomous point but rather as a microcosm constituted by the tropes and organizing figures characteristic of the episteme. For Foucault, individuals do not constitute culture; culture constitutes individuals. Moreover, the concept of the episteme implies that different sites within a given cultural period are self-similar. Foucault (1970) finds that during the age of classical reason, for example, the same organizing tropes appear in grammar, biology, political theory, and psychology.

A criticism frequently made of this approach is that it does not 
explain how changes take place through time (assume, for the purposes of this argument, that Foucault has characterized the periods accurately). How and why did people stop believing in similitude as an organizing trope and start believing in representation? The objection is not so straightforward as it appears, for what counts as a temporal explanation in the new paradigms is fundamentally different from what counts in the old. In traditional models, a temporal explanation placed groups of autonomous actors along a time line and advanced them according to laws or generalizations that explained why the actors behaved as they did. In the new paradigms, by contrast, temporal explanation connotes an understanding of the structural principles that relate different sites together by self-similarity, along with rules that state how these principles evolve over time. Foucault has achieved the first step in this kind of explanation by anatomizing the structural principles that underlie self-similar forms. Although he has not given a full temporal explanation, he has nevertheless come closer to it than his critics acknowledge, because they discount comparison of different kinds of self-similarities as the foundation on which temporal explanations are built.

Foucault's work suggests that the new paradigms could provide a model for culture, as well as for disciplines within the culture. Recall that in the sciences of chaos, the local designates the site within the global at which the self-similarities characteristic of the system are reproduced. Conceived as images of each other, local and global are related as microcosm is to macrocosm, although each level also contains areas so complex that they are effectively chaotic. Movement between levels is easy or possible only when the symmetries align. Configurations that replicate symmetries are ripe for change, because at this point the macroscopic properties of the system are extremely sensitive to microscopic perturbation. Applied to cultures, such a model does not require that all configurations within a given culture be self-similar; but it predicts that when enough of them are, initial perturbations will have large-scale effects. From the viewpoint of the cultural analyst, the model offers several advantages. Because they occur at multiple sites and many levels, characteristic symmetries are relatively easy to document; and because the system at this point is susceptible to dramatic and sudden changes, the changes that do occur are easy to recognize. The model gives us a way to 
understand how Foucault could be right in postulating a self-similar episteme for a given period, and still does not commit us to saying that knowledge everywhere in this period was organized according to these tropes (because there are always random variations) or that culture always works like this. In other words, it gives us a way to say that the local and global sometimes reproduce each other so that universals appear to run through the system, and sometimes not.

The logical next step is to wonder about the episteme of which this model is characteristic. There is a self-reflexive turn to this thought which makes it hard to think, for the model is used to explain how different cultural sites make up an episteme, and the episteme is used to explain how such models could arise at multiple sites within the culture. In other words, the mark of our episteme is that the concept of an episteme is a thinkable thought. The circularity of the proposition may explain why Foucault, especially in his earlier texts, emphasizes that one can never know the episteme in which one lives. By making this claim, he forecloses the self-referential circling that would otherwise engulf the episteme of epistemes.

Is it true that one's own episteme must remain on the dark side of knowledge? On occasion Foucault seems to imply that since the episteme is the ground of knowing, it cannot itself be known, at least not by those who stand upon it. However, this argument underestimates the heterogeneity of culture. ${ }^{3}$ Older epistemes do not disappear; rather, they continue as substrata valid for organizing restricted spheres of experience. When I drive to the store or play volleyball, for example, I am a Newtonian, never doubting that the laws of motion apply unequivocally. I know this not only intellectually but kinesthetically and intuitively. When I wonder why the price of gasoline has risen ten cents this week, I contemplate the complex mutual interactions most economically expressed through the field concept that also serves as a metaphor for global society. When I watch fractal forms being constructed on a computer screen, I am aware of yet another kind of thinking, distinct from the other two, with a wide domain of applicability to natural forms and complex systems. In what episteme do I live? Not in any single epis-

'In Foucault's later work, especially in vol. I of A History of Sexuality (1980a), it becomes clear that he sees earlier epistemes as forming the substrata of later periods. 
temology, but in a complex space characterized by multiple strata and marked by innumerable fissures.

Our episteme is visible to us, at least in part, because it is not homogeneous. Stratified through time and fissured into different sites, its formations are various enough to provide multiple perspectives. By standing at one perspective-for example, at the prospect offered by the sciences of chaos-one can see more clearly other perspectives, such as that of critical theory. Yet the metaphor also implies that different sites are underlaid by a common ground. The similarities between critical theory and the science of chaos justify the claim that they constitute a common epistemology, if not an episteme. At the same time, there are rifts that reveal significantly different assumptions at work, which is another way of saying that these disciplines are located at different sites.

The interplay between difference and similarity, critical speculation and scientific theory, brings us back to the central issue. Can any totalizing theory be true, when we know that our conception of the world is dependent on the local particulars of our lives? Are the claims of science to represent an objective reality justified, in the face of all we know about how linguistic, cultural, and physical factors affect our vision of the world? We saw in chapter 7 that Serres attempted to answer these questions by recognizing the difference between prediction and retrodiction. I will pursue another tack, arguing that we can adjudicate the issue by recognizing that reality cannot be identified in its positivity but must be inferred from the constraints applicable in a given situation.

Consider, for example, how conceptions of gravity have changed over the last three hundred years. Gravity is conceived very differently in the Newtonian paradigm in the general theory of relativity. For Newton, gravity was the result of mutual attraction between masses; for Einstein, it was the result of the curvature of space. One might imagine still other kinds of explanation, such as a Native American belief that objects fall to the earth because the spirit of Mother Earth calls out to kindred spirits in other bodies. But no matter how gravity is conceived, no viable paradigm could predict that when someone steps off a cliff, she will remain spontaneously suspended in mid-air. This possibility is ruled out by the nature of physical reality. Although the constraints that lead to this result are 


\section{The Figure in the Carpet}

interpreted differently in different paradigms, they operate universally to eliminate certain configurations from the range of possible answers. Gravity, like any totalizing idea, is always and inevitably a representation; our entire perceived world consists always and only of representations. Yet within the representations we construct, some are ruled out by physical constraints, others are not. ${ }^{4}$

This position should not be misconstrued as positivism. Positivism was part of a foundationalist movement that sought to establish an unambiguous connection between theory and observation, knowledge and perception. To remove ambiguity and to demonstrate the necessary nature of the connection between theory and observation, positivism turned to formalization. The purpose of formalization was to ensure that no subjective elements entered into the transformation of observation to theory. If one assumed that the scientific method reliably screened valid observations from suspect data, one then had a carefully forged chain that stretched from the foundation of facts to the superstructure of theory.

Today positivism is often evoked in literary studies as a synonym for naive realism. Its position with respect to realism is ambiguous, however, rather like the position of relativity theory with respect to classical physics. In one sense, relativity theory is the culmination of classical physics, because it is strictly causal and dedicated to preserving the universality of physical laws. In another sense, it marks a rupture with classical physics, for it demonstrates that the Newtonian paradigm is applicable over only a limited range of conditions. Moreover, it transforms the meaning of space and time even within the limited range that Newtonian mechanics covers. Similarly, in one sense positivism is an attempt to extend realism and make it more rigorous. In another sense, the very need to inject rigor indicates that realistic premises, by themselves, are shaky. Only when the chain from observation to theory is perceived to be weak is it necessary to strengthen it.

There is no need to rehearse here the well-known story of how and why positivism failed. Quantum mechanics, Gödel's theorem, and the sociology of science collaborated to show that causality in

'This argument obviously has certain affinities with Karl Popper's arguments regarding falsification (1965:33-65), although Popper is committed to a stronger version of realism than my position requires. 
the physical world is problematic, that formalization can never be complete, and that observations are always shaped by preexisting assumptions. The failure of foundationalist approaches across a range of disciplines led to an era of reflexivity, when artists, philosophers, writers, and scientists became fascinated by the paradoxical possibilities of a mind that watches itself thinking that it is thinking, of a left hand that draws a right hand drawing the left hand, of a text that writes a text about writing a text. This reflexive turn of mind is the parent, or at least the stepparent, of the vacuum that produces something out of nothing. The fecund vacuum would not be the powerful image it is in contemporary thought if reflexivity had not prepared the way by intimating that a lack of ground can be productive and exhilarating rather than threatening. Even if there are no foundations, there may nevertheless be creation, evolution, and renewal.

Consider how the position I articulated earlier compares with positivism. The proposition that we are always already within the theater of representation assumes at the outset that no unambiguous or necessary connection can be forged between reality and our representations. Whatever reality is, it remains unknowable by the finite subject. The representations we construct are determined by such historically specific factors as prevailing disciplinary paradigms and cultural assumptions, as well as by such fundamental givens as the human sensory apparatus and neurophysiology. Observations, then, are culturally conditioned and anthropomorphically determined. Thus we can never know how our representations mesh with reality, for we can never achieve a standpoint outside them.

Nevertheless, within the range of representations available at a given time, we can ask, "Is this representation consistent with the aspects of reality under interrogation?" If the answer is affirmative, we still know only our representations, not reality itself. But if it is negative, we know that the representation does not mesh with reality in a way that is meaningful to us in that context. The difference between a representation that is consistent with reality and one that depicts reality is the difference between a metaphor and a description. In this sense all theories are metaphoric, just as all language is.

The position I am describing abandons all attempts to arrive at foundationalist certainty. On the contrary, it grants that the connec- 
tion between reality and representation is essentially unknowable. It emphasizes that reality cannot be known in its positivity, only partially sensed through the failure of some representations to mesh with some aspects of reality. Because its thrust is toward explanations that are consistent with reality rather than reality itself, it could aptly be called tropism. Unlike positivism, it invites cultural readings of science, for the representations presented for disconfirmation have everything to do with prevailing cultural and disciplinary assumptions.

An omniscient viewpoint is clearly not appropriate to tropism, for the implication that reality can be seen completely and transparently is fundamentally at odds with the obscurity tropism posits between reality and representation. A better metaphor than vision is touch, for in contrast to sight's universalizing tendencies, touch is always localized.' Not only is it limited to specific body surfaces; it is also limited in the portions of an object it can apprehend. As the fable about the three blind men touching an elephant suggests, the difference between seeing and touching is the difference between a totalizing concept and competing local representations. We can say, then, that in our brushes with (not view or vision of) reality, it cannot be seen, but it can sometimes be felt as our representations push against the limits of the possible. From this position, we can grant the enabling premise of science, that reality can be felt; we can also grant the enabling premise of cultural analysis, that what we see is always and only a representation, never reality itself.

If such a common ground exists, why does contemporary critical theory so often see (or feel) itself opposed to science? Undoubtedly there are many reasons. One is the continuing imbalance between the power and prestige of science and those of other disciplines. As long as science is accepted as the arbiter of reality, other disciplines will resist by empowering their own inquiries at its expense. Also

'In "Towards a Critical Regionalism," in Foster, I983, Kenneth Frampton makes a similar proposal. He writes, "The liberative importance of the tactile resides in the fact that it can only be decoded in terms of experience itself: it cannot be reduced to mere information, to representation or to the simple evocation of a simulacrum substituting for absent presences" (p. 28). The claim that touch cannot be encoded as information is probably not correct; for example, Mandelbrot has proposed fractal dimensionality as a measure of surface roughness. But this is a quibble. The thrust of the comment, that touch has historically not been associated with abstraction, is true enough. 
important is the lingering of logical positivism within the scientific community. In the eyes of the logical positivists, what I have been calling the common ground is the muck of ambiguity; and as long as this muck is seen as something to be cleaned up through formalization rather than as a problematic to be explored, cultural analysts will see the scientific method as inimical to their projects. Still other factors are the differences in the criteria for publishable work in the two fields. It is possible to be a productive scientist and never seriously consider the problematics of representation. The situation is very different in contemporary critical theory, where questioning the nature of representation is virtually a necessity for work in the field.

As important as any of these factors, however, is the different roles that constraints play in the two fields. For literature, and to some extent for the human sciences, the important constraints are social rather than physical. ${ }^{\circ}$ For example, it would be possible to demonstrate that Hamlet is a dramatic text rather than a place mat or a name tag by refererence to the physical constraints that distinguish these forms. But of course no one is interested in making this kind of argument. For arguments people are interested in makingthat our interpretation of Hamlet is constrained by a community of readers, for example-the constraints are social rather than physical and hence are themselves social constructions. How does one define a community of readers? How does one know what attitudes they hold? Because of the social nature of literary constraints, it is tempting for literary theorists to believe that physical constraints are also constructions, and thus incapable of unambiguously indicating whether a physical theory is false.

"For this insight I am indebted to conversations with Mark Seltzer.

?Lately this position has also been argued by some philosophers of science, although it has not made much headway among scientists themselves. For a persuasive argument that experimental replication is a social construction, see H. M. Collins, Changing Order: Replication and Induction in Scientific Practice (Beverly Hills, Calif.: Sage, 1985). Also relevant is B. Barnes and D. Edge, eds., Science in Context: Readings in the Sociology of Science (Cambridge: MIT Press, 1982). Gabriel Stolzenberg carries the argument into mathematics by pointing out that the sense in which a proposition has been "proved" is dependent on prior and unexamined language usage, in "Can an Inquiry into the Foundations of Mathematics Tell Us Anything Interesting about Mind?" in Psychology and Biology of Language and Thought: Essays in Honor of Eric Lenneberg, ed. George A. Miller and Elizabeth Lenneberg, pp. 221-269 (New York: Academic Press, 1978). 
In my view, the difference between physical and social constraints lies in the fact that physical constraints manifest themselves in isomorphic ways in different representations, whereas social constraints are specific to the representation within which they occur. The present limit on silicon technology, for example, is a function of the speed with which electrons can travel through the semiconductor. One could argue that "electron" is a social construction, as are "semiconductor" and "silicon." There is an unavoidable limit inherent in this constraint, however, and it will manifest itself in whatever representation is used, provided it is relevant to the representational construct. Suppose that the first atomic theories had developed on the basis of the concept of waves rather than particles. Then we would probably talk not about electrons, semiconductors, or silicon but about indices of resistance and patterns of refraction. But there would still be a limit on the speed with which messages could be conveyed by means of silicon materials. If both sets of representations were available, one could demonstrate that the limit expressed through one representation was isomorphic with the limit expressed in the other.

Note that I am not saying constraints tell us what reality is. This they cannot do. But they can tell us which representations are consistent with reality and which are not. By enabling this distinction, constraints play an extremely significant role in scientific research, especially when the representations presented for disconfirmation are constrained so strongly that only one is possible. The art of scientific experimentation consists largely of arranging situations so that the relevant constraints operate in this fashion. No doubt there are always other representations, unknown and perhaps for us unimaginable, that are also consistent with reality. The representations we present for disproof are limited by what we can imagine, which is to say by the prevailing modes of representation within our culture. But within this range, invariable physical constraints can operate to select some as consistent with reality, others as not. The search for knowledge in the theater of representation thus proceeds by nay-saying rather than yea-saying. We cannot see reality in its positivity; we can only feel it through universal constraints operating upon local representations.

The representations that many scientists now find interesting are 
self-similar with the contemporary episteme, in the sense that they are themselves models of the aleatory and ambiguous connection between representation and reality. Focusing on complex systems that are inherently unpredictable, chaos theorists recognize that chance variations are intrinsic to these systems, and consequently that their representations will never coincide with the system's actual behavior. To this extent, chaos theorists are closer to contemporary critical theory than to Euclid, Newton, or Einstein. Yet there remain significant differences in emphasis between contemporary literature and science. To elucidate them, I turn now to the articulation of local knowledge within critical theory.

Local knowledge has become such an article of faith in the human sciences that it is paradoxically on the verge of becoming a universal in its own right. The transformation of local knowledge into global theory occurs as follows. First, the theorist insists that any attempt to create a global theory will be defeated by the intransigence of local sites that refuse to be co-opted into the global. Then he takes the further step of recognizing that this vision itself constitutes a global theory. Since it cannot be refuted through locality, it is the most successful and complete global theory possible. There is a growing inclination within literary circles to regard deconstruction in these terms, as a theory of local knowledge more totalizing than the totalizing theories it criticizes. ${ }^{8}$ Obviously, most of the people who make this observation are not deconstuctionists. In Paul de Man's seminal article "The Resistance to Theory" (I982), a similar argument is made by someone on the inside rather than the outside. The result is an extremely complex dialectic in which critic and discipline, local site and global theory, rhetoric and grammar, theory and resistance to theory become so intertwined that each simultaneously implies and repudiates the other.

The opposition that de Man places at the center of his text is the distinction between grammar and rhetoric. The essence of grammar, de Man says, is its universality. Grammar, like logic, must apply to many different texts and language usages, for if it did not, it would not have the force of rule we associate with it. Rhetoric, by contrast,

${ }^{8}$ See, for example, Wendell V. Harris, "Toward an Ecological Criticism: Contextual versus Unconditional Literary Theory," College English 48 (February 1986): I I 6-I 3 I. 
is unique to a given text. Tropes, in particular, "pertain primordially to language" and thus cannot be excavated from the language that constitutes them. In the act of reading, the "latent tension between rhetoric and grammar precipitates out," for grammar tries to account for rhetorical figures through extralinguistic generalizations that aim toward "mastering" the text. The attempts of grammatical readings to arrive at total accounts of the text are destined to be unsuccessful, however, for "there are elements in all texts that are by no means ungrammatical, but whose semantic function is not grammatically definable" (pp. 15-16; emphasis added).

Let us pause to think about this claim, for it acts as a pivot in de Man's argument. How does one prove that grammatically undecidable elements exist in all texts? Clearly it is not enough to give multiple examples, as de Man does so persuasively in Allegories of Reading (1979a), for the skeptic can always respond by saying that although the claim may be true for text $A$, text $B$, text $C, \ldots$, that does not mean it is so for all texts. This objection holds even if the examples number in the thousands, for as long as one text remains unexamined, it is possible that the generalization may be false. In fact, to make the claim in this strong universal form, one cannot use an empirical method. The only way to prove that the claim is true for all texts is to use a method analogous to a mathematical proof, that is, to limit oneself to the rules of logic and the assumptions implicit in the proposition in order to prove that the conclusion is irrefutable. Of course de Man does no such thing, for the good reason that no one could do it. Propositions of this kind are not susceptible to proof in the mathematical sense.

Here you may object that I am putting too much weight on a careless overstatement, a momentary slip of the pen. Were the article to end with the claim that grammar will always be defeated by the resistance of undecidable rhetorical elements, the objection would be well taken. The claim would be worth making even if it were true only in a certain number of cases and hence would not depend on universality for its force. The rest of the article, however, makes clear that the claim's universality is at the heart of what de Man means by "resistance to theory" and thus is central to the argument as a whole. De Man wants rhetoric not only to resist grammar but to resist it irresistibly. Rhetoric can unequivocally defeat totalizing 
grammars only if it is always able to resist; and this ability requires that rhetoric, symbol and figure of resistance, harbor within itself a generalization, namely, the universal claim at issue.

Hence de Man concludes that

technically correct rhetorical readings may be boring, monotonous, predictable and unpleasant, but they are irrefutable. They are also totalizing (and potentially totalitarian) for since the structures and functions they expose do not lead to the knowledge of an entity (such as language) but are an unreliable process of knowledge production that prevents all entities, including linguistic entities, from coming into discourse as such, they are indeed universals, consistently defective models of language's impossibility to be a model language. [De Man, I982: 20; emphasis added]

For de Man, to recognize the universality of rhetoric's defeat of grammar is to recognize that rhetoric as a theory of reading is able to resist every universalizing claim except its own universality. The more successful the theory is, the more its insistence on locality is transformed into a kind of universality, so that "the language it speaks is the language of self-resistance." True to his theory, de Man ends by emphasizing that not all locales can be subsumed even into theory's resistance to itself, for "what remains impossible to decide is whether this flourishing is a triumph or a fall" (p. 20). To end with such a poignant and paradoxical interpenetration of the local and global shows what a consummate master of rhetoric de Man was.

But wait. How did we arrive at this point again? Through the claim that there are rhetorical elements in all texts which resist grammatical coding. And where did this claim come from? Not from any method of proof that would force us to the "ineluctable necessity" of recognizing its truth (p. I 7 ), but from the assertion of the writer. The necessity, in other words, must be located within the writer's psychology rather than within the nature of the case itself. What makes this conclusion so curious is that at the beginning of the article de Man writes with great scorn about the psychological aspects of teaching. "Overfacile opinion notwithstanding, teaching is not primarily an intersubjective relationship between people but a cognitive process in which self and other are only tangentially and contiguously involved. The only teaching worthy of the name is 
scholarly, not personal" (p. 3). De Man makes clear that for him scholarship is an escape from psychology, not an exhibition of it. As in the return of the repressed, psychology nevertheless comes back, for it is only through psychology that we can understand the springs of that "ineluctable necessity" to forge a global theory of local knowledge. Knowing, then, that if de Man were alive he would detest the argument $I$ am about to make, I will make it anyway in the hope of understanding not just personal quirks in his writing but larger patterns of thought characteristic of the ideology of local knowledge. ${ }^{9}$

The article is so cunningly structured that, to do it justice, I will need to look closely at how it reaches the crux of locating the resistance to theory within theory itself. In the opening paragraphs, de Man sketches in the following context. He had been asked to write the article on theory for the Modern Language Association volume Introduction to Scholarship in Modern Languages and Literatures. Clearly what the MLA committee had in mind was a survey of the field which would identify major trends and lay out the important issues. Instead, de Man wrote an article (presumably the one we are reading, or a draft of it) which valorized only one theory-his version of rhetorical reading-and characterized other kinds of theory as either nontheory or muddled resistance to theory. It is scarcely surprising that the MLA committee declined to use the article in their volume. De Man says he "thought their decision altogether justified, as well as interesting for its implications for the teaching of literature" (p. 3). What are those implications? For de Man, the teachable is the transmissible, and the transmissible is the generalizable. But if the essence of literariness is the nongeneralizable, "then scholarship and teaching are no longer necessarily compatible" (p. 4). Immediately, then, the conflict between the local and global is set up as a central dialectic. At the beginning its focus is de Man versus the MLA committee, or more generally scholarship versus teaching,

'This chapter was written before de Man's reviews in Le Soir surfaced. The questions these reviews raise about de Man's inclination toward totalizing theories are no doubt relevant to my argument; but since my interest is in the turns that de Man's argument takes rather than his theories as such, I have chosen not to consider the reviews. 
but it soon moves inward in tightening spirals until finally it locates the conflict within theory itself.

These spirals are constructed through a series of oppositional stances that are debates about what theory ought to do versus what theory is actually able to do. Theory ought "in theory" (p. 4) to begin by defining its object; but it is unable to do so because until recently, it has always relied on extrinsic systems ("philosophical, religious or ideological”) for its internal organization (p. 5). Thus defeated, theory may as well start pragmatically. This observation leads de Man into a survey of New Criticism, which was a movement not because it was theory but because it was uniformly hostile to theory. What, then, is theory? "Literary theory can be said to come into being when the approach to literary texts is no longer based on non-linguistic, that is to say historical and aesthetic, considerations" (p. 7). Specifically, theory comes into existence with the advent of structural linguistics, for then it is based not on an extrinsic system but on the structure of language itself.

Locating the basis for literary theory within language is an ambiguous move, for while it "gives language considerable freedom from referential restraint," it also "makes [language] epistemologically highly suspect and volatile, since its use can no longer be said to be determined by considerations of truth and falsehood, good and evil, beauty and ugliness, or pleasure and pain" (p. Io). And in fact, this "autonomous potential of language," which de Man identifies with literariness, makes literary theory intensely threatening to some people. Those who raise objections to this view of language are, however, not worth answering, for their arguments are "always misinformed or based on crude misunderstandings" (p. I 2). Thus dismissing as unworthy opponents those who resist "real theory" because they disagree with structuralist views of language, de Man locates the only meaningful resistance to theory within theory itself-and so arrives at the crux discussed above.

If we think about how this argument is constructed, we can see that it proceeds as a series of inward-turning movements, always in the hope of finding a position strong enough to be defended, an opponent worthy enough to act as a serious contender. Theory becomes real theory when it locates its basis within language; resis- 
tance becomes real resistance when theory resists itself; reading becomes real reading when it resists de Man's theory of reading. Finally we are left with the spectacle of de Man resisting de Man, for he can find an opponent powerful enough to be interesting only within his own constructs. He is certain that this is a flourishing, but whether it "is a triumph or a fall" remains undecidable. It is undecidable because at this point local knowledge is indistinguishable from global theory, just as de Man is indistinguishable from the activity of reading. As local site merges with global system, the psyche looks at its object but sees only another version of itself, in an endless mirroring of self-similarities.

Let us not forget, however, that this inward turning of the theory toward paradoxical self-similarity hinges upon the universal claim that is the crux of the argument. If the theory is not pushed to this limit-if one is content to say that some or even most texts are configured so as to have grammatically undecidable elements-then the paradox does not appear, for then one is simply saying that not all sites can be accounted for by the universal theory in question. Why does de Man want to push the local into the global? There may be a clue in the subtle irony with which he uses the word "mastery." "Mastery," in de Man's usage, connotes universalizing moves made by opponents to limit the scope and power of rhetorical theory. Stanley Fish is said to have written a "masterful" essay when he tries to "empty rhetoric of its epistemological impact" (p. I9); grammar aims toward "mastering" meaning when it tries to subsume rhetorical figures in grammatical codes (p. I6). When de Man creates a global theory of local knowledge, he simultaneously repudiates and practices mastery in this sense, for he resists totalization by totalizing. Mastery is intolerable because it is identified with totalitarianism; but it is also unavoidable, because the only way always to resist totalizing moves is through a theory more universally applicable than what is resisted. The ideology of local knowledge, pushed to the extreme, is thus inextricable from the totalitarian impulses it most opposes. The unflinching honesty with which de Man faces this paradox is admirable, for it implies a profound awareness that impulses toward mastery are still masterful even when they are directed against mastery. The dream of local knowledge, that it can 
always and everywhere defeat global theory, carries within it its own subversion.

Most theorists working with local knowledge do not push its claims as far as de Man does, and the paradoxes that are the crux of de Man's argument consequently do not appear. But the very excessiveness of his argument, in an article widely cited in the profession, indicates how much the current of our time runs toward local knowledge. Even in scientific disciplines with strong traditional orientations toward global theory, local knowledge has come sufficiently to the fore to make the local/global dialectic problematic in ways it was not previously. In humanistic fields, the bias toward local knowledge is stronger. Making global arguments now in literary criticism is akin to trying to write psychobiography during the heyday of the old New Criticism. No one actually prevents such arguments from being made; but the prevailing practices of the discourse community tend so strongly in contrary directions that anyone who attempts them feels an invisible pressure pushing against each paragraph, each sentence, each word. Surely it is significant that a thinker such as Michel Serres, whose mind seems naturally drawn toward the abstract and global, chooses to deploy his abstractions in favor of local knowledge. ${ }^{10}$ When Serres explicitly entertains the question whether his work is local or global, he invariably represents it as local although it partakes at least as much of global abstraction. A similar point could be made about Foucault's representation of his work in comparison with the work itself.

What in the present cultural moment makes the case for local knowledge seem so compelling? Why has it come to the foreground in such diverse projects as the New Historicism and nonlinear dynamics, fractal geometry and social archaeology, rhetorical criticism and strange attractors? What does it signify for our culture, our discourses, and our future that we are currently much more inclined toward of diversity than homogeneity, particularity than generality,

\footnotetext{
"Maria Assad has convincingly argued that in Serres's later work, he turns increasingly toward the local, repudiating the impulse toward globalization which marked his earlier essays. See Maria Assad, "Michel Serres: In Search of a Tropography," in Chaos and Order: Complex Dynamics in Literature and Science, ed. $\mathrm{N}$. Katherine Hayles (Princeton University Press, forthcoming).
} 

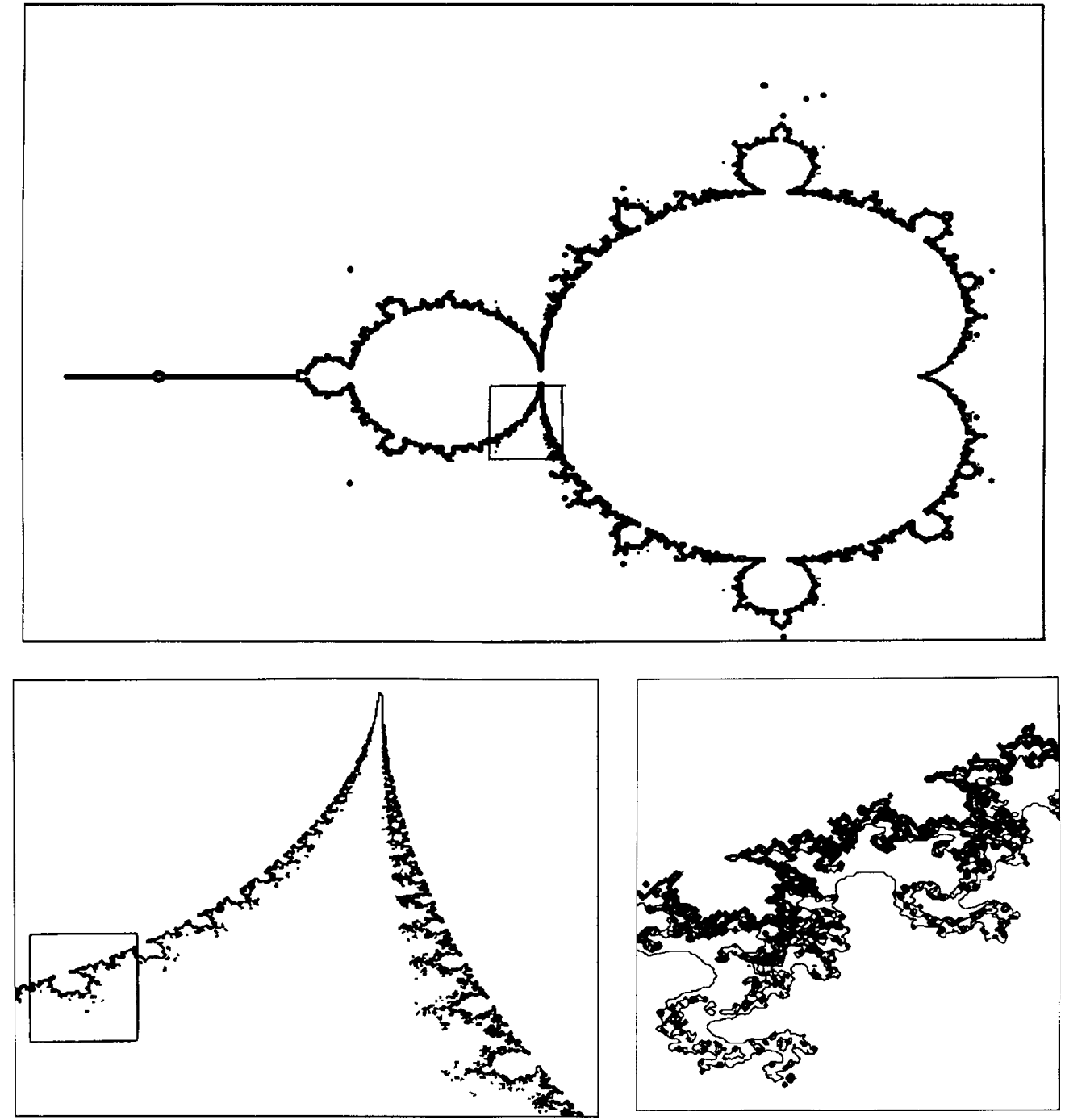

9. Mandelbrot set with progressively greater magnifications of boxed areas. Courtesy of Nural Akchurin. 
refusal of mastery than mastery? The next chapters explore these questions in relation to Lessing's Golden Notebook and to postmodernism.

Meanwhile, from the information technology with which our future seems entwined are emerging images that I offer as correctives to the ideology of local knowledge. I am thinking of the haunting and evocative computer simulations of complex systems which show regions of overall symmetry intermixed with asymmetric hazy regions. When one of the hazy regions is magnified, it shows a mixture of symmetry and haze; and when one of the hazy regions of this magnified portion is magnified, it continues to show a mixture of symmetry and haze (figure 9). The image never resolves to either order or chaos, continuing to show infinitely fine-grained complexity for as long as magnification proceeds. Is not this an apt image for the interplay between the local and the global?

Laplace dreamed of an infinitely predictable world, but chaos intervened. De Man dreamed of a theory of representation that could not be universalized, but this in itself was a universal. Although global theory and local knowledge may each attempt to deny the other by extending its boundaries, when one is pushed to the limit, the other returns. Local knowlege and global theory are as much partners in the creation of representation as order and chaos are in the creation of the world. For as far as we can see or feel, clarity and haze, local variation and global form, intermingle. 